\title{
Laboreal
}

Volume $1 \mathbf{N}^{\circ} 1$ | 2005

Varia

\section{La actividad psico-educativa-sanitaria de las maestras de preescolar en guardería, una relación de servicio específica}

A actividade psico-educativa-sanitária das educadoras do préescolar no jardim de infância, uma relação de serviço específica

L'activité psycho-éducative-sanitaire des professionnelles de la petite enfance en crèche, une relation de service spécifique

Psycho-educative-sanitary activity of pre-school teachers in kindergarten : a specific service relation

\section{Rafael González}

\section{OpenEdition}

\section{Journals}

Edición electrónica

URL: http://journals.openedition.org/laboreal/13956

DOI: $10.4000 /$ laboreal. 13956

ISSN: 1646-5237

\section{Editor}

Universidade do Porto

\section{Referencia electrónica}

Rafael González, « La actividad psico-educativa-sanitaria de las maestras de preescolar en guardería, una relación de servicio específica », Laboreal [En línea], Volume 1 №1 | 2005, Publicado el 01 diciembre 2005, consultado el 20 octubre 2019. URL : http://journals.openedition.org/laboreal/13956 ; DOI : 10.4000/laboreal.13956

Este documento fue generado automáticamente el 20 octubre 2019.

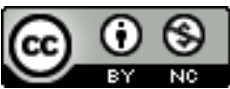

Laboreal está licenciado com uma Licença Creative Commons - Atribuição-NãoComercial 4.0 Internacional. 


\section{La actividad psico-educativa- sanitaria de las maestras de preescolar en guardería, una relación de servicio específica}

A actividade psico-educativa-sanitária das educadoras do préescolar no jardim de infância, uma relação de serviço específica

L'activité psycho-éducative-sanitaire des professionnelles de la petite enfance en crèche, une relation de service spécifique

Psycho-educative-sanitary activity of pre-school teachers in kindergarten : a specific service relation

Rafael González

\section{NOTA DEL EDITOR}

Manuscrito recibido en : septiembre/2005

Aceptado tras peritage en : noviembre/2005

\section{Introducción : Comprender las actividades de servicio psicoeducativas-sanitarias en guardería}

1 El objetivo de este texto es caracterizar las actividades de servicio con finalidad psicosanitaria-educativas, con relación a "otras relaciones de servicio" con un objetivo comercial o administrativo. El interés proviene por un lado, por la cantidad de investigadores y consultores, pertenecientes a distintas disciplinas, en comprender las 
relaciones de servicio. Y por el otro, el abordaje psico-ergonómico, propuesto en ésta investigación, efectuado en una guardería de la ciudad de Paris en Francia.

2 En general, las actividades de servicio fueron definidas por Hill (1977) "como la transformación de la condición de un individuo, de un bien u objeto que pertenece a otro". Delaunay et Gadrey (1987), modelaron esta actividad como triangular : "realizada por un prestador $\mathrm{A}$, que intenta transformar una realidad $\mathrm{C}$, perteneciente y solicitada por B".

3 Este proceso se encuentra enmarcado en un "contexto técnico de relación moral y de confianza" (Goffman, 1968), "que incluye acciones dirigidas a las personas, con una finalidad social y sanitaria" (Gadrey, 1996, 2002). Esto último, permite señalar que desde un punto de vista organizacional, el proceso de prestación de servicio, se inscribe en un sistema institucional o familiar que define las reglas de intervención. En fin, la actividad desarrollada en estas estructuras o instituciones, están impulsada por actores y orientada hacia polos y no simplemente una relación triangular.

4 En las guarderías, el prestador de servicio (maestras de preescolar) se divide en planificador y ejecutor, ya que este define los objetivos y las reglas de trabajo proceso enmarcado en la protección y el seguimiento, que en este caso son niños.

5 Los solicitantes se dividen en :

- Usuarios externos, (padres o representantes), en contraposición con un cliente, ya que la contribución financiera que le es solicitada está asociada al ingreso del grupo familiar ;

- Beneficiarios (los niños), ya que estos no se encuentran en una relación puntual con los prestadores (maestras de preescolar), sino en un proceso donde las acciones de estas, persiguen mejorar y garantizar las condiciones de vida, orientar la conducta social y psicológica, así como de educarlos (Dubet, 2002).

6 En un sistema como este, las tareas cotidianas del personal se encuentran en la interfase de reglas organizacionales, estructurales, las solicitudes de los usuarios y las necesidades de los beneficiarios. Desde un punto de vista psico-ergonómico, y asociado a las 35 horas, así como a la reorganización de la prestación del servicio, en este estudio se consideró la actividad de vigilancia, las tareas interferentes y la regulación de las maestras de preescolar, como proceso cognoscitivo esencial para el cumplimiento de la misión en esta ocupación.

\subsection{Estudio de la vigilancia de las maestras de preescolar en guardería : una aproximación psico-ergonómica}

7 Con el objeto de responder a la misión compartida por la estructura, los beneficiarios y los usuarios, las maestras de preescolar, ejecutan al menos cuatro tareas fundamentales :

- la vigilancia,

- los cuidados sanitarios,

- el intercambio de información con los padres, e

- intercambio de información entre maestras.

De estas cuatro tareas, la vigilancia a los beneficiarios, con un objetivo de protección, se visualiza como la tarea que ocupa la mayor parte del tiempo de las maestras y fundamental para iniciar cualquier acción verbal o no verbal. 
9 Tradicionalmente, en ergonomía, el análisis de las actividades de vigilancia se han realizado sobre el control de los procesos dinámicos, tales como los de la industria de la petroquímica (Rocher, 1982), los hornos industriales (Hoc, 1991), la industria nuclear, el transporte (Cellier, 1985), el pilotaje de avión (Amalberti, 1996). De forma general, lo que ocurre desde un punto de vista cognoscitivo es que los trabajadores construyen una representación de la situación, a fin de establecer el diagnóstico que permita garantizar eficacia, eficiencia, fiabilidad, seguridad y protección del sistema, así como garantizar su propia salud y la de otros.

10 Estas situaciones se caracterizan por el hecho que pueden evolucionar más o menos rápidamente, sin la acción directa de los trabajadores. Lo que implica una actividad de identificación de fallas de funcionamiento de las maquinas o de las instalaciones; considerando información y parámetros dados por instrumentos o dispositivos de visualización de información, (Leplat, 1968 ; Leplat \& Chesnais, 1973), así como prever, detectar esos cambios, evaluar los riesgos y controlar la situación, en un período temporal mas o menos corto, incluso bajo presión (Amalberti, 1996). Se trata entonces de una actividad de regulación de situaciones adversas que puedan ocurrir. Esas regulaciones pueden efectuarse de diferentes formas, por anticipación o de forma reactiva, según las circunstancias y las experiencias de las personas involucradas (Faverge, Olivier, Delahaut, Stephaneck \& Falmagne, 1966); en la espera de una señal o buscando activamente la información (De Montmollin, 1997). Todas ellas suponen el conocimiento de variables técnicas, temporales y espaciales en juego.

11 Sin lugar a dudas, la gran mayoría de éstas características, son en parte transferibles al trabajo de las maestras de preescolar en guardería. El trabajo de éstas consiste en recabar información que les permita diagnosticar que algo esta inadecuado o que existe la probabilidad de riesgos y que deben actuar ante las múltiples señales enviados por los niños con una intensidad variable y diferente (gritos, llantos, sonidos...), según el conocimiento y la experiencia de la trabajadora, la vigilancia puede ser reactiva o preventiva.

12 Basándonos en los resultados citados, se puede hacer la hipótesis que una estrategia de control de anticipación (preventiva) puede ser más eficaz en cuanto a la calidad del servicio prestado, que respondiendo a las urgencias o de forma reactiva. Sin embargo, esta vigilancia puede tener interferencia con las otras actividades de las maestras de preescolar (cuidados sanitarios, intercambio de información con los padres y puesta al día de eventos nuevos entre maestras). De hecho, se puede preguntar si la vigilancia preventiva es siempre realizable en caso de que el equipo de maestras de preescolar por sección este incompleto.

\subsection{Gestión de tareas interferentes de las maestras de preescolar}

La principal característica de las cuatros tareas que ejecutan las maestras de preescolar, es que estas pueden competir entre si por diferentes razones :

- Los niños pueden tener actividades o necesidades diferentes al mismo momento ;

- Los padres van y vienen al momento del recibimiento en la mañana y en la tarde y informan o solicitan información sobre el estado, evolución o comportamiento de los hijos, pero las maestras deben, al mismo tiempo, vigilar los niños que ya han llegado, a la sección.

14 No obstante, en situación de tareas múltiples, el sujeto debe compartir su atención entre varias fuentes de información y tiene varias tareas simultaneas que deben ser 
ejecutadas bajo presión temporal (Leplat, 1982). Si consideramos la distinción o noción de Ouni (1998), las maestras de preescolar pueden estar confrontadas a dos tipos de situación de trabajo compartido: múltiples e interferentes. En el primer caso, el momento de aparición de las tareas es previsible ; la trabajadora tiene la posibilidad de planificar u organizar las diferentes tareas. Esto es lo que puede ocurrir en el transcurso de períodos ya establecidos o rutinarios en la guardería (recibimiento en la mañana y en la noche, juegos pedagógicos, almuerzos, meriendas...).

En el segundo caso, el sujeto se encuentra confrontado al surgimiento imprevisto de tareas, que necesita bien sea interrumpir la tarea que se estaba realizando, bien sea asegurar de forma simultánea con la que está ejecutando (Gadbois, 1981; Gadbois, Logeay, \& Maline 1988). Esto se puede ilustrar en el caso de exigencias o solicitudes de diversos niños o de adultos, que la maestra de preescolar debe gestionar en un tiempo relativamente corto y al interior de otros tiempos dedicados a otra actividad. De hecho, la gestión temporal de tareas interferentes es definida "como la escogencia entre varias posibilidades en función de objetivos eventualmente cambiantes y del contexto de la situación" (Cellier, 1996).

Desde un punto de vista cognoscitivo, los trabajadores actúan como reguladores del funcionamiento del sistema, utilizando medios, formas de acción o de regulación diferentes para gestionar los recursos, con el objeto de solventar los riesgos de perturbación con compromisos que solo persiguen alcanzar la misión o la tarea prescrita (Valot, Weill-Fassina, Guyot \& Amalberti, 1996). Esos compromisos se traducen por variaciones de comportamiento intra e inter individuales, que solo persiguen satisfacer diversos criterios que aseguren un equilibrio entre la práctica, las exigencias y las solicitudes.

Los estudios ergonómicos que se describen a continuación ofrecen excelentes descripciones de tales regulaciones en el caso de tareas interferentes :

- Anticipación de interrupciones en el control de trafico de bus (Cellier, 1985);

- Determinación de la urgencia de la solicitud y definiendo un orden de prioridad (Gadbois, 1981), en un centro de vigilancia y de información (Ouni \& Weill-Fassina, 1998). En este último caso, de tareas independientes y donde el operador no tiene la iniciativa, se puede distinguir diferentes tipos de gestión de la tarea interferente :

- § Ignorada ; la tarea no es tratada,

§ Inmediata ; la tarea es tratada de forma prioritaria,

§ Diferida ; la tarea es realizada luego de la tarea de referencia,

- Simultanea ; la tarea interferente y de referencia son tratadas al mismo momento,

§ Alterna; cuando la herramienta es informatizada.

Solicitando una nueva distribución de tareas con los colegas : en un servicio neonatal de noche, Barthe (1999) muestra este fenómeno en detalle; en unos talleres de bobinas (Mariné \& Navarro, 1980) ; en un centro de urgencia medical (Benchekroun, 1984) ; y en caso de situación perturbada (Filippi, 1994).

Todas las estrategias, tanto individuales como colectivas, dependen de la experiencia de los trabajadores. Notablemente, un principiante, podría tener tendencia a responder inmediatamente frente a la urgencia, a dejarse guiar por los eventos exteriores, a anticipar poco y a considerar las urgencias según como van apareciendo (Gaudart \& Weill-Fassina, 1999). 

experiencia, una maestra de preescolar en la guardería se deje llevar por los eventos exteriores (presencia de los padres, de los colegas, o de los gritos de alarmas lanzados por los niños) y efectuar en consecuencia, una vigilancia más reactiva. Por el contrario, una maestra con mucho mas experiencia pondría en marcha un proceso de vigilancia más preventiva y organizaría de forma más precisa su trabajo en función de las urgencia y las tareas.

Es posible que esas diferentes estrategias permitan hacer frente con una mayor o menor eficacia y eficiencia, a las situaciones en las cuales falte personal por las ausencias no remplazadas, como es el caso de este estudio, donde por razones de la reducción de la jornada de trabajo de 7.5 horas contra la amplitud de horario de la guardería $12 \mathrm{~h}$ las maestras ingresaban en forma intercalada, desde las $7 \mathrm{~h} 30$ hasta las 10h00, pero donde la totalidad de los niños ingresaba a las 8h30, 20 niños por sección.

El interés de este estudio consistió en analizar las exigencias a las cuales las maestras de preescolar de una guardería debían hacer frente, y cuales son las estrategias puestas en marcha con el objeto de asegurar la calidad del servicio esperado. Todo esto enmarcado en una estrategia de privilegiar el ingreso de las maestras menos experimentadas a las $7 \mathrm{~h} 30$ de la mañana y las más experimentadas a finales de la mañana : $10 \mathrm{~h} 00$.

\section{Metodología : Una aproximación cuali-cuantitativa para comprender el trabajo de maestras de preescolar en guardería}

En ésta aparte se describirá las modalidades de recolección de los datos, así como los elementos consideradores para dar cuenta de la actividad de regulación de las maestras de preescolar en una sección de bebés.

\subsection{Escogencia de las situaciones y personas analizadas}

La sección de bebés fue seleccionada por dos razones :

$\S$ La ausencia de personal (vacaciones que no son asumidas por otra persona externa) genera mas dificultades y riesgos a los niños, ya que el nivel de autonomía y de comprensión de la palabra es incipiente y además porque estos necesitan mucha manipulación ;

$\S$ Se encuentra constituida por dos unidades de edades diferentes :

- Unidad A, con niños de edades entre 11 y 14 meses ;

- Unidad B, con niños de edades entre 3 y 10 meses.

Esta diferencia de edades es uno de los factores que condicionan la organización de actividades durante el día y el año, organización que intenta satisfacer las necesidades individuales de los niños que se encuentran en pleno crecimiento.

El momento del recibimiento fue seleccionado, por un lado, porque se constató que durante este período, las maestras siempre estaban incompletas con respecto a la norma que señala : una auxiliar de preescolar por cada cinco niños presentes en la sección de bebés. Por el otro, este es un momento, donde la presencia de los padres, con 
los cuales se intercambia información, pueden interferir con la tarea esencial de vigilancia de las maestras.

\subsection{Principios generales de recolección de los datos}

\section{efectuadas con la ayuda de una cámara de video. Las principales ventajas de est} técnica son :

- Permitió el análisis de múltiples aspectos de una situación dinámica en la cual varios actores interactúan,

- Posibilidad de observar, el número de veces tanto que fue necesario, para seleccionar la secuencia más pertinente para el propósito de este estudio,

- Permitió, analizar los datos desde varios puntos de vista y verificar su validez,

- Ofreció la posibilidad de intercambio o de entrevistas reflexivas con los interesados. dialogo y luego transcritas en su integridad. Estas fueron agrupadas en unidades de pocos minutos, con el objeto de describir los eventos, las interacciones y las acciones. esquema orientador para analizar lo grabado. Se distinguió particularmente : $\S$ El tipo de acción :

- Acciones verbales : definidas como todo el enunciado de las maestras de preescolar,

- Acciones no verbales: definidas como toda otra acción no verbale de las maestras de preescolar : exploración visual, desplazamiento, gestos con la cara,

- A quien iba dirigido la acción : niños, padres u otras maestras de preescolar,

§ El objetivo de la acción :

- Preventivo : definida como toda acción verbal o no verbal de anticipación de las maestras dirigido a los niños,

- Reactivo : definida como toda acción de reacción de las maestras, ante los comportamientos individuales o grupales, de los niños,

- Informativo : definida como toda acción verbal o no verbal de solicitud o transmisión de información relacionado con los niños, dada a los padres

$2^{\mathrm{a}}$ fase : Categorización del tipo de actividad

Luego de haber realizado los primeros análisis y con el objeto de comparar las secuencias que fueron analizadas, las acciones verbales y no verbales fueron clasificadas según tres tipos de actividades, antes definidas: vigilancia, cuidados sanitarios e intercambio de la información entre padres y maestras de preescolar, con el objetivo preventivo o reactivo.

38 A partir de las tareas y las hipótesis efectuadas al final del numeral 1.1. y 1.2. se definieron varios tipos de caso, efectuados por las maestras de preescolar en las guarderías :

- Vigilancia preventiva : anticipación de los riesgos de peligro de la integridad corporal con relación a los objetos y otros niños. 
- Vigilancia reactiva : reaccionar frente a un peligro a la integridad corporal, con relación a los objetos y otros niños.

- Cuidados sanitarios preventivos : anticipar un evento en los niños con el objeto de asegurar el confort físico y psicológico,

- Cuidados sanitarios reactivos: reaccionar frente a un comportamiento, un olor, una observación o un evento en los niños, con el objeto de asegurar el confort físico y psicológico,

- Intercambio de información con los padres : proceso de dialogo de información entre las maestras de preescolar y los padres, relativo a los niños,

- Puesta al día de información e intercambio de información entre maestras de preescolar : proceso de dialogo de información y coordinación de actividades concerniente a los niños.

\subsection{Las secuencias analizadas}

Las secuencias analizadas permitieron caracterizar las maestras de preescolar por :

- Los tipos de tareas realizadas,

- Los tipos de actividad,

- La importancia de las actividades,

- Definir los criterios de comparación de las actividades de vigilancia y de las tareas interferentes, durante el recibimiento en las mañanas en la sección de bebés.

- Las maestras de preescolar observadas, participaron de forma voluntaria en el estudio; y para filmar a los niños se solicitó la autorización de los padres.

Con el objeto de considerar el conjunto de acciones efectuadas por las tres maestras de preescolar, durante el recibimiento de los bebés en la mañana entre $7 \mathrm{~h} 30$ y $10 \mathrm{~h} 00$, diez secuencias de trabajo se analizaron para en un total de 120 minutos. Para evidenciar la repartición temporal de las actividades, seis secuencias fueron seleccionadas, de una duración de 6 minutos, permitiendo comparar las diferentes situaciones de trabajo, éstas características permitieron comparar la gestión en diversas situaciones según la experiencia.

41 Como se explicó en la parte inicial de este artículo, el interés de comparar estas operadoras, perseguía obtener elementos objetivos para reorganizar el recibimiento de los beneficiarios y usuarios externos de la guardería en cada sección, considerando la experiencia y el número de niños que llegan entre las 7 h30 y las 8 h30, así como el número de maestras de preescolar presentes, en contraposición a lo que se hacía en la guardería, de considerar solo la reducción del tiempo de trabajo de 35 horas y la amplitud de horario de la guardería. Es evidente que esta comparación exploratoria es insuficiente para generalizar los resultados a otras situaciones de servicio, pero útil para dar cuenta de la complejidad de esta ocupación en el contexto del estudio y sobre todo a la similitud de organización que tienen la mayoría de las guarderías en Paris. En fin las variables independientes consideradas fueron :

43 § La experiencia profesional :

- Una debutante (1 año en esta sección),

- Una antigua (11 de experiencia en esta sección),

- Una debutante suplente (1 año en esta sección),

$\S$ El número de maestras de preescolar presentes en la sección durante el recibimiento en la mañana : una y/o dos, lo que corresponde al incumplimiento de la tasa de paridad 
de las maestras de preescolares con respecto a los niños. En ninguna de las secuencias analizadas esta tasa de paridad de una maestra por cada cinco niños, fue respetada, por una u otra razón. En las secuencias analizadas en detalle, las maestras o se encuentran solas o a dos para un número superior a la tasa establecida de niños, diferentes actividades en la gestión de las tareas en las diferentes situaciones. Estas comparaciones solo tienen un valor indicativo, susceptible de evidenciar las hipótesis plausibles. En efecto, y considerando las exigencias del campo de investigación, estos resultados solo corresponden al comportamiento de tres maestras de preescolar de una guardería durante los meses de Abril, Mayo y Junio.

\section{Resultados: Importancia de las actividades según la configuración de las situaciones de recibimiento en la mañana}

La importancia de las diferentes actividades por todas las situaciones incluidas

La Tabla 2 presenta por cada tipo de actividad, el número de acciones efectuado por las

\subsection{Una carga de trabajo elevada} tres maestras de preescolar durante el recibimiento. De esta tabla se puede señalar :

Durante los 120 minutos de grabación, 1305 acciones fueron contabilizadas, lo que quiere decir una media de 3,5 acciones diferentes/minuto ejecutada por maestras de 
preescolar, lo que es relativamente importante y pudiera explicar al menos la impresión de las maestras de efectuar una trabajo en serie y de no hacer un buen trabajo.

Tabla 2 : Número de acciones, por tipo de actividad efectuada para el total de las tres maestras de preescolar durante 120 min., mientras efectuaban el recibimiento en las mañanas en la sección de bebés.

\begin{tabular}{cccc} 
Tipo de actividad & Acciones verbales & Accionesno verbales & Total \\
\hline Vigilancia & 180 & 476 & 656 \\
\hline Cuidados sanitarios & 79 & 160 & 239 \\
\hline $\begin{array}{c}\text { Intercambio de } \\
\text { información con los }\end{array}$ & 120 & 40 & 160 \\
padres & & & \\
\hline $\begin{array}{c}\text { Puesta al dia de } \\
\text { situaciones nuevas } \\
\text { entre las maestras de } \\
\text { preescolar e } \\
\text { intercambio de } \\
\text { información }\end{array}$ & 124 & 126 & 250 \\
\hline Total & & 1305
\end{tabular}

\subsection{Actividades que exigen una atención compartida entre beneficiarios, usuarios y colegas}

Las acciones son en su gran mayoría no verbales, dirigidas a los niños (cargar en los brazos, besos, colocarlos en una posición corporal más segura, cambio de pañales), una gran cantidad de acciones verbales dirigida a los padres (intercambio de información) y combinación de acciones verbales y no verbales, orientadas hacia los colegas (intercambio de información, miradas para identificar que hacer). Estas reparticiones dejan suponer tareas cumplidas de manera simultánea o alterna. Las interacciones y coordinaciones entre maestras de preescolar bajo acciones verbales y no verbales, representan cerca de un quinto de las actividades y constituyen cualitativamente la segunda actividad más importante, confirmando así la existencia de un trabajo colectivo a corto plazo.

\subsection{La vigilancia como actividad esencial}

Las acciones no se reparten igualmente entre los diferentes tipo(s) de actividad : más de un tercio de entre ellas, son dirigidas hacia los niños; la vigilancia representa aproximadamente la mitad del número total de acciones observadas. La vigilancia de los niños y los cuidados sanitarios dejan aparecer una ligera tendencia de acciones con orientación preventiva (Tabla 3). En ese medio, donde la noción de riesgo es importante $\mathrm{y}$ donde muchos incidentes fueron constatados, el número de acciones reactivas, 
orienta a preguntarse sobre las modalidades de estrategia de gestión de tareas interferentes según la configuración del recibimiento.

Tabla 3 : La orientación de las acciones dirigidas hacia los niños.

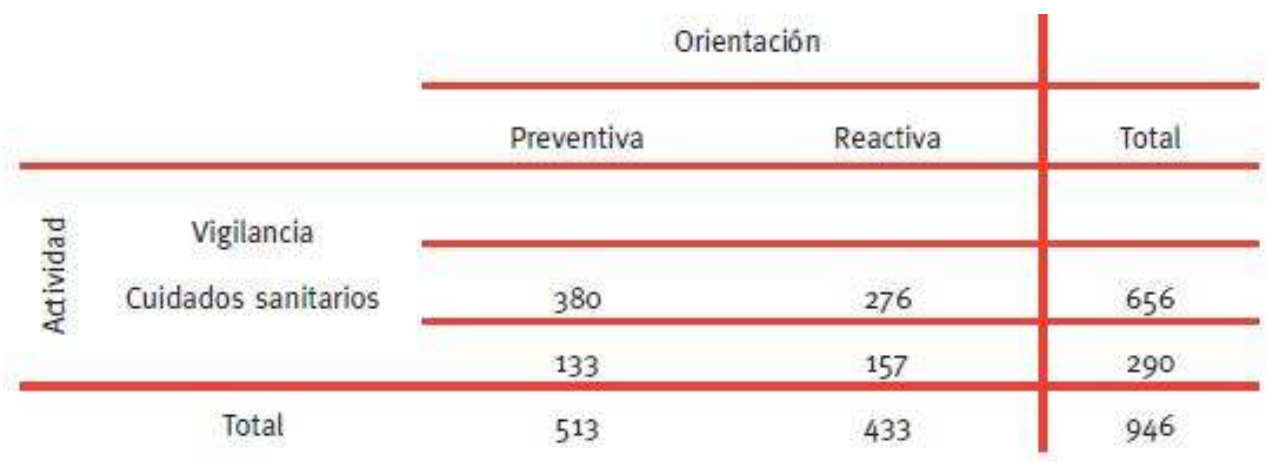

\subsection{Una gestión diferenciada de las actividades.}

La comparación de diferentes secuencias es ordenada según la situación que se supone la menos perturbada (dos maestras sin padres) a la situación la más perturbada (una maestra con dos padres). La duración total en segundo y el número de acciones que fueron utilizados para la comparación responde a una escogencia metodológica y no a una evaluación de la performance de las maestras, dado que el servicio esperado es efectuado de manera diferente. En el caso donde hay dos maestras, se utilizó el tiempo total y el número de acciones de una sola maestra, sea la principiante o la antigua, según la secuencia analizada.

\subsection{Duración y número de acciones efectuadas por dos maestras durante el recibimiento, con padres ausentes (secuencia IV y VI)}

Las maestras principiantes y antigua se encuentran acompañadas con una maestra suplente, pero ningún padre se encuentra presente en la sección, el número de los niños es de 14 en cada secuencia IV y VI. El objetivo de este análisis es comparar los modos de gestión de las tareas interferentes entre maestra fija y suplente, la vigilancia y los cuidados sanitarios, orientados hacia los niños y realizadas por la maestra antigua, principiante y suplente. 
Figura 1 : Comparación de la duración de las acciones en segundos de una maestra principiante y una antigua ayudada por una maestra suplente, sin la presencia de padres (Secuencia IV y VI).

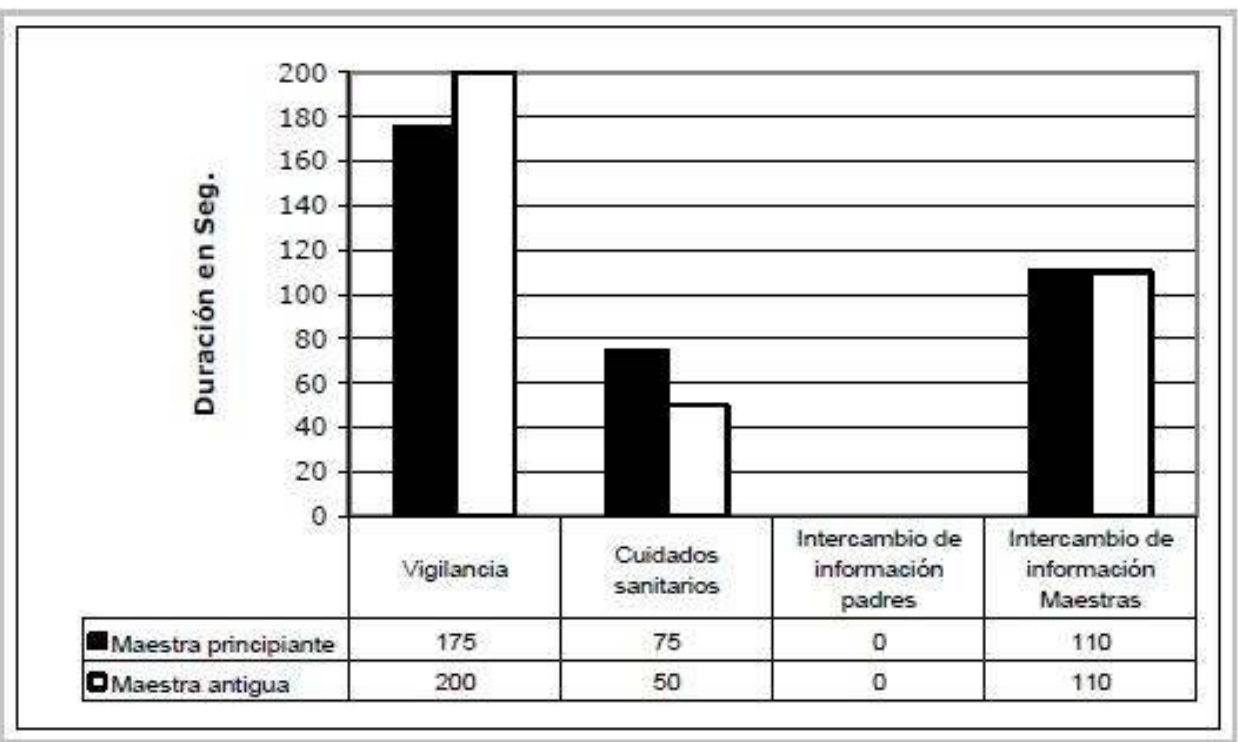

Tabla 4 : Duración en segundo y número de acciones de una maestra principiante y una antigua, ayudadas ambas por una maestra suplente y padres ausentes.

\begin{tabular}{|c|c|c|c|}
\hline \multicolumn{4}{|c|}{ Secuencia IV } \\
\hline Maestra principiante & Duración media en seg. & Número de acciones & Duración total en seg. \\
\hline Vigilancia & 7 & 25 & 175 \\
\hline Cuidados sanitarios & 5 & 15 & 75 \\
\hline $\begin{array}{l}\text { Intercambio de infor- } \\
\text { mación entre maes- } \\
\text { tras }\end{array}$ & 10 & 11 & 110 \\
\hline Total & & 51 & 360 \\
\hline \multicolumn{4}{|c|}{ Secuencia VI } \\
\hline Maestra antigua & Duración media en seg. & Número de acciones & Duración total en seg. \\
\hline Vigilancia & 10 & 20 & 200 \\
\hline Cuidados sanitarios & 5 & 10 & 50 \\
\hline $\begin{array}{l}\text { Intercambio de infor- } \\
\text { mación entre maes- } \\
\text { tras }\end{array}$ & 10 & 11 & 110 \\
\hline Total & & 41 & 360 \\
\hline
\end{tabular}

Luego de la lectura de la Tabla 4 y la Figura 1, correspondiente a las secuencias IV y VI, se puede constatar que :

- La vigilancia es la actividad esencial, seguida del intercambio de información y de interacciones entre las maestras, los cuidados sanitarios llegan en tercer lugar. El tiempo total de las acciones es equivalente al tiempo de grabación, lo que implica que las actividades fueron efectuadas en serie una detrás de la otra. 
- El número de acciones efectuadas por las maestras principiantes es ligeramente más elevado, con respecto a la maestra antigua; sobre todo lo relativo a la vigilancia y los cuidados sanitarios y análogos para el intercambio de información entre maestras.

- Sin embargo, si la duración en segundo del conjunto de actividades de la maestra antigua y de la principiante es idéntica, la distribución del tiempo que se le da a los niños es diferente ; el tiempo de vigilancia es mas elevado y el tiempo de cuidados sanitarios es menor.

Estos resultados se explican de forma más cualitativa por una distribución diferente de actividades según la maestra :

- Con la principiante, es la maestra suplente que cambia de pañal a los niños y los acuesta para la siesta, ella sale completamente de la sala de recibimiento, mientras que la otra se ocupa en la sala de otras acciones sanitarias que garantizan el confort de los niños.

- Con la antigua, hay una distribución equitativa del conjunto de los cuidados sanitarios, lo que deja más tiempo dedicado a la vigilancia.

\subsection{Duración y número de acciones efectuadas por dos maestras con padres presentes (secuencia II y IV)}

La Tabla 5 y Figura 2, presentan una pareja de maestras, principiante fija y principiante suplente, en interacción con algunos padres y 19 niños presentes en la secuencia II ; así como en la secuencia IV, una pareja de maestras, antigua y principiante suplente, con 18 niños presentes. El objeto de esta comparación es mostrar la gestión de tareas interferentes realizadas por las maestras y el impacto de la presencia de los padres con respecto a las situaciones precedentes.

Figura 2 : Comparación de la duración de las acciones en segundos de una maestra principiante y una antigua, ayudadas por una maestra suplente, con padres presentes (Secuencia II y IV).

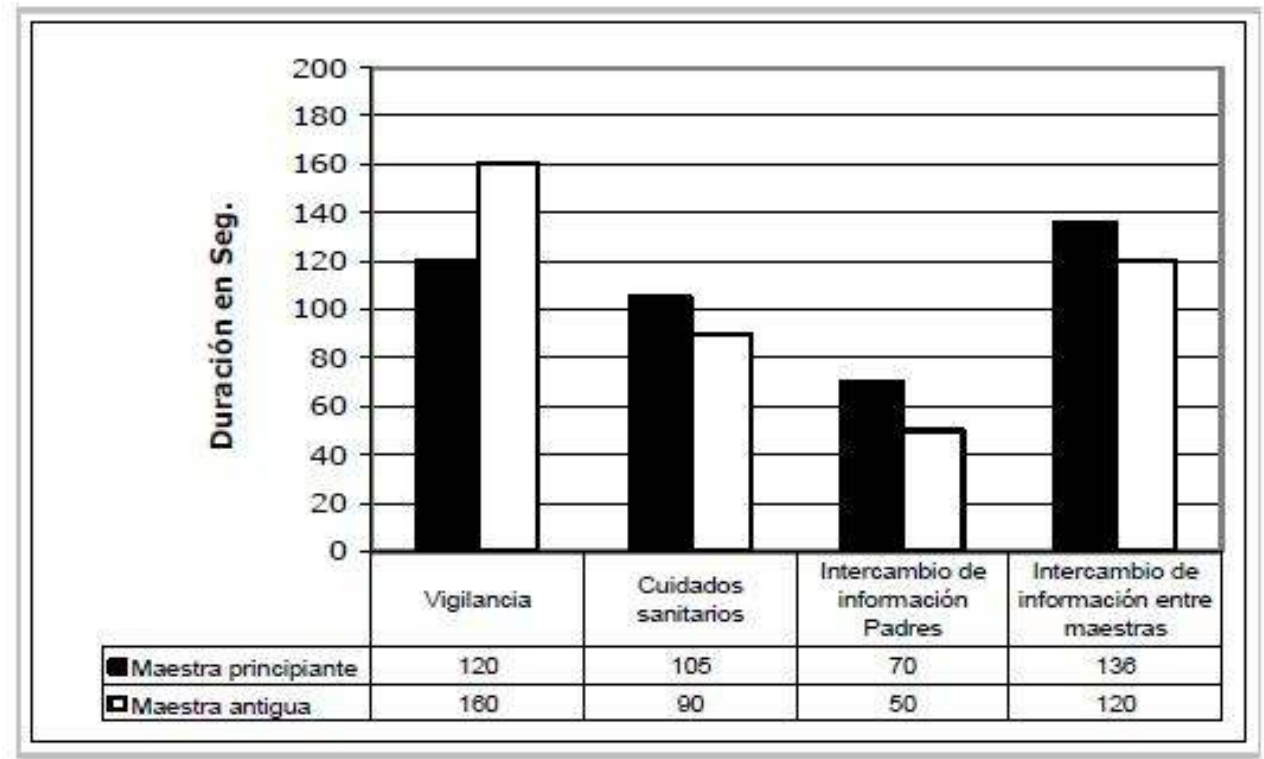


Tabla 5 : Duración en segundos y número de acciones de una maestra principiante y una antigua, ayudadas por una maestra suplente $y$ en presencia de padres.

\begin{tabular}{|c|c|c|c|}
\hline \multicolumn{4}{|c|}{ Secuencia II } \\
\hline Maestra Principiante & Duración en seg. & Número de acciones & Total de Seg. \\
\hline Vigilancia & 6 & 20 & 120 \\
\hline Cuidados Sanitarios & 5 & 21 & 105 \\
\hline $\begin{array}{c}\text { Intercambio de } \\
\text { información con los } \\
\text { padres }\end{array}$ & 7 & 10 & 70 \\
\hline $\begin{array}{l}\text { Intercambio de } \\
\text { información entre } \\
\text { maestras }\end{array}$ & 17 & 8 & 136 \\
\hline Total & & 59 & 431 \\
\hline \multicolumn{4}{|c|}{ Secuencia IV } \\
\hline Maestra Antigua & Duración en seg. & Número de acciones & Total de Seg. \\
\hline Vigilancia & 8 & 20 & 160 \\
\hline Cuidados Sanitarios & 10 & 9 & 90 \\
\hline $\begin{array}{c}\text { Intercambio de } \\
\text { información con los } \\
\text { padres }\end{array}$ & 10 & 5 & 50 \\
\hline $\begin{array}{c}\text { Intercambio de } \\
\text { información entre } \\
\text { maestras }\end{array}$ & 20 & 6 & 120 \\
\hline Total & & 40 & 420 \\
\hline
\end{tabular}

En la tabla 5 y figura 2, correspondientes a las secuencias II y IV, se constata que :

En función del tiempo consagrado a cada actividad, el orden de las prioridades es análogo: vigilancia, cuidados sanitarios, intercambio de información con la maestra suplente e interacción con los padres. Esta última actividad se suma bien a otras actividades, mientras que en el caso precedente el tiempo total de las acciones era igual al tiempo de grabación ; aquí el tiempo total de las acciones es superior, lo que traduce un modo de gestión simultanea de las diferentes actividades.

El número de acciones de la maestra principiante es aproximadamente de un tercio más elevado que el de la maestra antigua. Sin embargo, la importancia dada a los niños en vigilancia y cuidados sanitarios aparece de manera más remarcada en la antigua.

2 Además, la duración media de las diferentes actividades de la maestra antigua, es más importante con respecto a de la maestra principiante. Lo que significa un tiempo mas largo consagrado a cada acción.

3 La repartición de las acciones, la duración y la división de las actividades con la suplente, orienta a pensar que la maestra principiante se deja más llevar por exigencias externas a la vigilancia que la antigua.

4 Con respecto a la repartición de las actividades, como ya se describió, es la maestra principiante suplente, en la secuencia II, que realiza los cuidados sanitarios, cambia los pañales de los niños, los mete en la cama y recibe eventualmente los padres. Por el contrario, en la secuencia IV, la maestra antigua realiza una distribución equitativa de las tareas materiales y del recibimiento de los niños y los padres. 


\subsection{Duración y número de acciones efectuadas por una maestra con padres presentes (secuencia I y III)}

La Tabla 6 y la Figura 3 presentan las secuencias I y III, perteneciente al trabajo de una maestra principiante y una antigua, con padres presentes y 10 niños en la secuencia I y 12 niños en la secuencia III. El objetivo de esta comparación es mostrar el impacto de la triple “perturbación" de la situación sobre las actividades: 1 maestra, 3 padres presentes y número de niños por encima de lo establecido en la norma.

Figura 3 : Comparación de la duración de las acciones en segundos de una maestra principiante y una antigua, solas en presencia de padres.

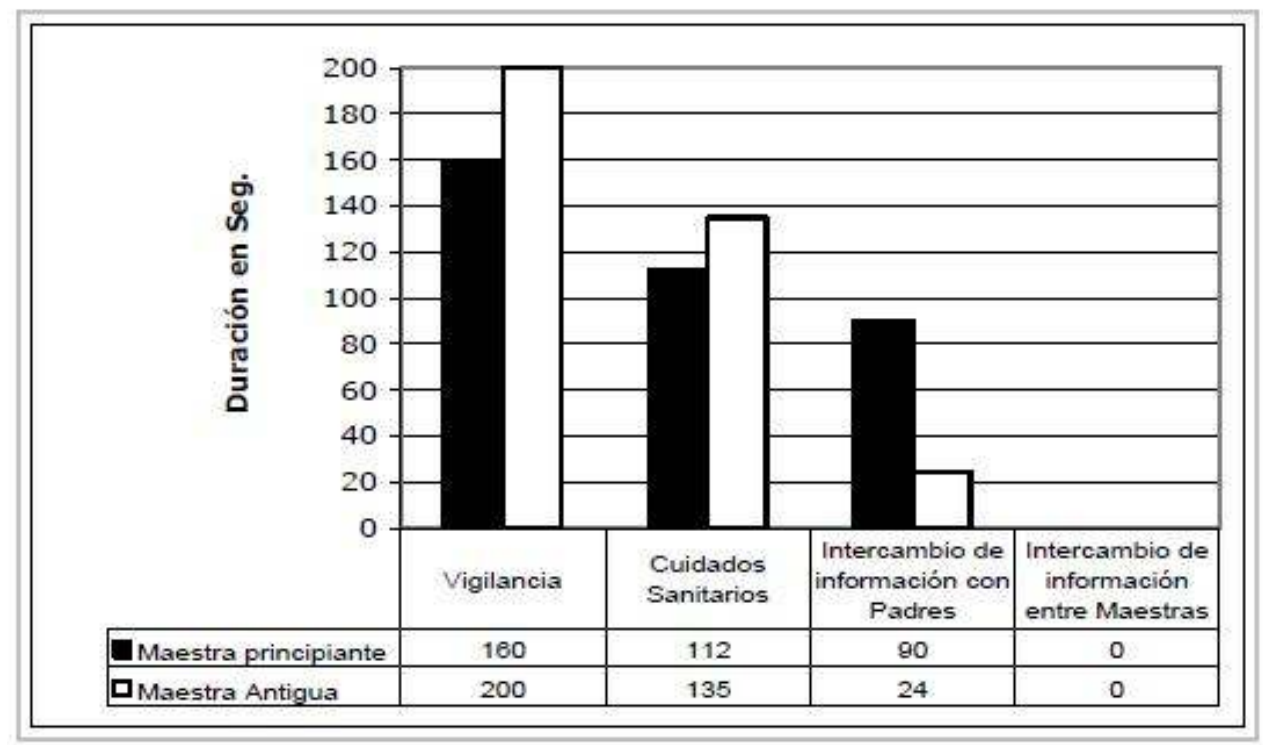


Tabla 6 : Duración en segundos y número de acciones de una maestra principiante y de una antigua, cada una sola, y con presencia de padres.

\begin{tabular}{|c|c|c|c|}
\hline \multicolumn{4}{|c|}{ Secuencia I } \\
\hline Maestra Principiante & $\begin{array}{c}\text { Duración en } \\
\text { segundos }\end{array}$ & Número de acciones & Total en seg. \\
\hline Vigilancia & 5 & 32 & 160 \\
\hline Cuidados Sanitarios & 7 & 16 & 112 \\
\hline $\begin{array}{c}\text { Intercambio de } \\
\text { información con los } \\
\text { padres }\end{array}$ & 15 & 6 & 90 \\
\hline Total & & 54 & 362 \\
\hline \multicolumn{4}{|c|}{ Secuencia III } \\
\hline Maestra Antigua & $\begin{array}{c}\text { Duración en } \\
\text { segundos }\end{array}$ & Númerode acciones & Totalen seg. \\
\hline Vigilancia & 8 & 25 & 200 \\
\hline Cuidados Sanitarios & 9 & 15 & 135 \\
\hline $\begin{array}{c}\text { Intercambio de } \\
\text { información con los } \\
\text { padres }\end{array}$ & 6 & 4 & 24 \\
\hline Total & & 44 & 359 \\
\hline
\end{tabular}

- En las dos secuencias y considerando la duración y el número de acciones, la vigilancia es la actividad principal, seguido de los cuidados sanitarios, luego el intercambio de información con los padres,

- El número de acciones efectuadas por la maestra principiante es superior aproximadamente de un quinto en comparación a la maestra antigua.

- Como en el primer caso, el tiempo total es prácticamente el mismo de la grabación, lo que traduce una gestión sucesiva de las actividades,

- La duración de la vigilancia sigue siendo prioritaria, pero la atención a los cuidados sanitarios se encuentra aumentada con respecto a los casos precedentes, lo que confirma el papel asignado a la maestra suplente,

- La diferencia más remarcable entre la maestra antigua y la principiante, es la atención prestada a los padres, reducida a lo mínimo en número y en duración por la primera, al preferir en consecuencia las actividades dirigidas a los niños. Al limite, la gestión de esta actividad es casi apartada por la maestra antigua, mientras que esta es tratada de forma inmediata o simultánea por la principiante.

\subsection{La comparación de estas tres situaciones evidencian :}

- Una división de la actividad más importante en la maestra

- principiante que en la antigua,

- Una vigilancia prioritaria en las dos maestras principiantes, pero en la maestra antigua es mas elevado que en las principiantes,

- Actividades de cuidados sanitarios más frecuentes en las principiantes que en la antigua, solo en los casos cuando están solas. La ayuda de la maestra suplente, pareciera que se orienta sobre todo hacia las actividades que aumentan en frecuencia, y ausencia de la compañera, 
- Un recibimiento de los padres que es considerado como una tarea interferente, pero al mismo tiempo necesaria, sin embargo en la maestra antigua, pareciera que disminuyen los intercambios y en su duración,

- Si se compara la repartición de actividades en las tres situaciones, la antigua modula la gestión, ya que esta respeta sus prioridades, mientras que la principiante los distribuye de forma igual y se deja llevar por los eventos externos.

- La prolongación de la duración media de las acciones en la maestra antigua, pareciera ser un síntoma de un dominio de las actividades interferentes. Esto se traduce a nivel cualitativo ; al visionar las secuencias video, se observó una aparente lentitud en las acciones, es decir una impresión de control y dominio de la situación, inclusive se evidencia por la tranquilidad de los niños alrededor de la maestra antigua que se coloca en el centro del tapiz y los niños se ubican a su alrededor.

- Entre otros aspectos, esta última también deja hablar a los padres y les responde poco, anota en el mismo momento las información(es) intercambiada(s) (y no luego) en el cuaderno de intercambio de información con las otras maestras. Esta información global se confirma durante las observaciones sistemáticas del desarrollo de las actividades en diferentes situaciones, pero no descritas en este documento.

\section{Discusión: Impacto de las situaciones perturbadas durante el recibimiento en guardería} situaciones perturbadas, se identifican tres modos de comportamiento :

- Carga ligera : se observa una orientación aproximadamente de $70 \%$ de las acciones dirigidas hacia los beneficiarios (niños) y un $30 \%$ de intercambio de información con los colegas,

- Carga media : se observa una estabilidad aproximativa de porcentajes de acciones dirigida hacia los beneficiarios (niños) (69 y $73 \%$ ), y un intercambio de acciones orientadas hacia los padres (17 y $15 \%$ ), así como los colegas (13 y $14 \%$ ) tanto por la maestra principiante que por la antigua.

- Carga elevada : sin colegas, se observa un comportamiento diferenciado. La principiante dirige $75 \%$ de sus acciones hacia los niños y un $25 \%$ hacia los padres. La Antigua orienta el $90 \%$ de sus acciones hacia los niños y un $10 \%$ hacia los padres. Si el porcentaje de las acciones orientadas hacia los niños es relativamente estable, el aumento de la carga de trabajo es marcada por una aceleración del ritmo de las acciones sucesivas, evidenciado por una anticipación importante de la principiante suplente y también por la aparición de acciones simultaneas y posteriormente un cierre de las actividades en la sala de la sección de bebés; las otras tareas son apartadas $\mathrm{u}$ olvidadas cuando la maestra principiante se encuentra sin ayuda de una compañera.

En esta relación de servicio se muestra como las maestras gestionan los compromisos entre los beneficiarios, los colegas y los usuarios y hacen frente a las situaciones perturbadas. Si la vigilancia y los cuidados sanitarios es la preocupación principal, cualquiera sea la situación, la maestra antigua pareciera que resiste y hace frente de la mejor manera a las perturbaciones. Es evidente que no se puede generalizar este resultado en las condiciones de observación, pero estos encuentran al menos algunas respuestas en otros estudios vinculadas con el desarrollo de competencia, específicamente lo concerniente a las respuestas de solicitudes exteriores del propio trabajo, en este caso, (intervención de los padres) ; en fin, en la gestión temporal de las 
actividades, la extensión del campo de acción y el trabajo colectivo (Lichtenberger, 1999 ; Weill-Fassina \& Pastre, 2004).

La repartición de las actividades con la maestra "suplente" no se efectúa de la misma manera :

- Sin la presencia de los padres, la maestra antigua intercambia información y delega ciertas acciones a la principiante, sobre todo consideradas como materiales (cambiar pañales y acostar los niños). Esta distribución del trabajo entre maestras puede ser calificada como "co-acción en la medida que las trabajadoras efectúan acciones diferentes, pero integradas a corto plazo en una actividad común"(Leplat \& Savoyant, 1984; Savoyant, 1985; Falzon, 1995 ; Grosjean \& Lacoste, 1999 ; De la Garza \& Weill-Fassina, 2000),

- En presencia de los padres, con una carga elevada, esta coacción se transforma en colaboración, es decir en una actividad colectiva en la cual las trabajadoras cumplen sobre un mismo "objeto" operaciones diferentes que se articulan las unas con las otras con un objetivo común a corto y mediano plazo (Barthe, 1999; Cosnier, Grosjean, \& Lacoste 1993; De la Garza \& Weill-Fassina, 2000).

En el fondo, en esta repartición, hay probablemente por parte de los auxiliares un conocimiento de las propias competencias, así como de las suplentes. Lo que muestra este resultado, es que la distribución de las tareas puede igualmente estar asociada a las características de los trabajadores, tales como la edad, la experiencia y el estado fisiológico (Daguet, Heddad, Rabit, \& Wallet, 2002). Los resultados de este estudio, son muy útiles para justificar una tasa de paridad mucho más dinámica en las guarderías ; considerando los padres, las actividades de las maestras, el crecimiento de los niños, y sobre todo el marco temporal y espacial en las guarderías.

\section{Conclusión : Múltiples niveles de decisión y de acción a gestionar}

70 En la sección de bebés, el análisis muestra que las situaciones perturbadas tienen un impacto sobre las condiciones de trabajo de las maestras de Preescolar y en la calidad del servicio prestado a los niños y padres.

Durante el recibimiento, la carga de trabajo se manifiesta no solamente por un número elevado de acciones y diálogos por minuto, sino también par numerosas tareas interferentes que se distribuyen entre vigilancia y cuidados sanitarios dirigida hacia los niños, y eventualmente de intercambio a los padres. Esto supone que las maestras de preescolar conocen las posibilidades de actuar, conocen sus propios recursos individuales, que en definitiva les permite estimar las posibilidades de dominar la situación (Chatigny, 2001). Esto pudiera explicar que, en ciertas configuraciones de situaciones, las maestras prefieren una estrategia más que otra, puesta en marcha con el objeto de evitar la sobre carga o el fracaso frente a los beneficiarios, las compañeras de trabajo, los padres y ellas mismas (Hoc, 1991).

El trabajo de las maestras de preescolar en este tipo de establecimiento aparece como un caso específico de relación de servicio, si se compara con otras que tienen una finalidad administrativa o comercial. Esta se desarrolla en el dominio del ser, ya que lo que intentan los operadoras es garantizar el cuidado sanitario y el desarrollo de cada niño, atendido individualmente pero considerado en el marco de un grupo (González, ClaireLouisor \& Weill-Fassina, 2001). 
En fin, la calidad del servicio prestado a los usuarios y los beneficiarios, dependen en cierta manera a la capacidad de regulación y dominio de las maestras de preescolar en gestionar las condiciones externas e internas de la actividad y sus efectos (Leplat, 1992; González, 2004 ; Gonzalez \& Weill-Fassina, 2005).

\section{BIBLIOGRAFÍA}

Amalberti, R. (1996). La conduite de système à risque. Paris : PUF.

Barthe, B. (1999). Gestion collective de l'activité de travail et variation de la vigilance nocturne : le cas d'équipes hospitalières en postes de nuit longs. Thèse de doctorat, Université Toulouse II, France.

Benchekroun, H.T., (1984). Modélisation et simulation des processus intentionnels d'interlocution. Application à la conception d'un système d'aide à la communication multi agents. Thèse de doctorat, Conservatoire National des Arts et Métiers, Paris, France.

Cellier, J.M. (1985). Stratégie de surveillance sur écran dans une tâche de régulation de trafic de bus. In R. Patesson, Ergonomie et Condition de travail en informatique, Bruxelles.

Cellier, J.-M. (1996). Exigences et gestion temporelle dans les environnements dynamiques. In JM. Cellier, V. De Keyser \& C. Valot (Eds.), La gestion du temps dans les environnements dynamiques (pp. 20-48). Paris : PUF.

Chatigny, C. (2001). La construction de ressources opératoires : contribution à la conception des conditions de formation en situation de travail. Thèse de Doctorat en Ergonomie, Conservatoire National des Arts et Métiers, Paris.

Cosnier, J., Grosjean, M., \& Lacoste, M. (1993). Soins et communication. Approches interactionnistes des relations de soins. Ethologie et Psychologie des communications. Lyon : PUF.

Daguet, I., Heddad, N., Rabit, M., \& Wallet, M. (2002). La place du sujet dans la relation de service à l'hôpital. In F. Hubault (Coord.). La relation de service, opportunités et question nouvelles pour l'ergonomie (pp. 120-146). Toulouse : Octarès Editions.

De la Garza C., \& Weill-Fassina, A., (2000). Régulations horizontales et verticales du risque. In T.H. Benchekroun \& A. Weill-Fassina (Coord.) Le travail Collectif. Perspectives actuelles en ergonomie (pp. 218234). Toulouse : Octarès Editions.

De Montmollin, M. (1997). Vocabulaire de l'ergonomie. Toulouse : Octarès. (2e édition revue et augmentée).

Delaunay, J.-C., \& Gadrey, J., (1987). Les enjeux de la société de service. Paris : Presses de la Fondation Nationale des Sciences Politiques.

Dubet, F. (2002). Le déclin de l'institution. France : Seuil.

Falzon, P. (1995). Les activités de service. In M. Montmollin (Ed.) Vocabulaire de l'ergonomie (pp. 218-220). Toulouse : Octarès Editions. 
Faverge, J-M., Olivier, M., Delahaut J., Stephaneck P., \& Falmagne J.C. (1966). L'ergonomie des processus industriels. Etudes de psychologie sociale et industrielle. Université Libre de Bruxelles : L'Institut de Sociologie.

Filippi G. (1994). La construction collective de la régulation du trafic RER. Thèse de doctorat, Université Paris-Nord, Paris, France.

Gadbois C. (1981). Les tâches interférentes, un problème caractéristique du travail hospitalier. Soins, $26,12,15-18$.

Gadbois, Ch., Logeay, P., \& Maline, J. (1988). Medical schedules and time managements in nursing. Stress and organisational problems in Hospitals : implications for management. In D.Wallis \& de Ch.J. Wolff (Eds.) London : Croom Helm.

Gadrey, J. (1996). L'économie des services. France : La Découverte. Gadrey, J. (2002). L'émergence d'un modèle du service : enjeux et réalités. France : Liaisons.

Gaudart, C., \& Weill-Fassina, A. (1999). L'évolution des compétences au cours de la vie professionnelle : approche ergonomique. FormationEmploi, (Activités de travail et dynamiques des compétences, $\mathrm{n}^{\circ}$ spécial), 67, 47-62.

Goffman, E. (1968). Asiles. Études sur les conditions sociales des malades mentaux. Paris : Ed. de Minuit.

González, R. (2004). Du macro au micro : les régulations des relations de service en crèches. Thèse de Doctorat en Ergonomie, Laboratoire d'Ergonomie du CNAM, Paris, France.

Gonzaléz, R., \& Weill-Fassina (2005) Modalités de régulation du processus de travail dans les activités de service en crèche. @ctivités, 2,2. www.activites.org

González, R., Claire-Louisor, J., \& Weill-Fassina, A. (2001). Les activités psycho-socio-éducatives : une catégorie spécifique de relation de service. Actes du Congrès de la SELF, (3-4-5 octobre), MontréalCanada.

Grosjean, M., \& Lacoste, M. (1999). Communication et intelligence collective. Le travail à l'hôpital. Paris : PUF.

Hill, P. (1977). On Goods and services. The review of income and wealth, 4, 315-338.

Hoc, J-M. (1991). Effets de l'expérience des opérateurs et de la complexité de la situation dans la conduite d'un processus continu à long délai de réponse : Le haut fourneau. Le travail humain,54, 3,225250 .

Leplat, J. (1968). Attention et incertitude dans les travaux de surveillance et d'inspection. Paris, Dunod (Sciences du comportement, $\left.n^{\circ} 6\right)$.

Leplat, J. (1982). Les situations de travail, terrain d'avenir pour la psychologie, in P. Fraisse (Ed), Psychologie de demain (pp. 311-330). Paris : PUF.

Leplat, J. (1992). Ergonomie et activités collectives. Actes du XXVIIe Congrès de la Self : Les aspects collectifs du travail, Lille, Septembre.

Leplat, J., \& Chesnais M. (1973). Le contrôle des produits industriels : Travaux actuels et perspectives d'études. Le travail humain, 3, I, 75-94.

Leplat, J., \& Savoyant, A. (1984). Ordonnancement et coordinations des activités dans les travaux individuels et collectifs. Bulletin de psychologie, 37, (364), 271-278.

Lichtenberger, Y. (1999). Compétence, organisation du travail et confrontation sociale. Formation Emploi (Activités de travail et dynamiques des compétences, $n^{\circ}$ spécial), 67, 93-107. 
Marine, C. \& Navarro, C. (1980). Ròle de l'organisation informelle du travail en équipe lors d'un dysfonctionnement technique. Bulletin de psychologie, Tome XXXIII, 344, 311-316.

Ouni, R. (1998). Modalités de gestion temporelle des tâches interférentes dans le contrôle de processus indépendants. Thèse de doctorat, École Pratique des Hautes Etudes, Paris, France.

Ouni, R., \& Weill-Fassina A. (1998). L'informatisation, une aide à la gestion des tâches interférentes. Actes du XXXIIe congrès de la SELF. Lyon. 233-243.

Rocher, M. (1982). Les activités de contrôle de processus partiellement automatisées dans une installation de fabrication biochimique. Diplôme d'ergonomiste. Conservatoire National des Arts et Métiers, Paris, France.

Savoyant, A. (1985). Conditions et moyens de la coordination interindividuelle d'opérations d'exécution sensorimotrices. Le travail humain, 48, (1), 59-72.

Valot, C., Weill-Fassina A., Guyot, S., \& Amalberti, R. (1996). Vers un modèle pour l'analyse ergonomique des grands systèmes, In : Ergonomie et production industrielle, L'homme dans les nouvelles organisations, Actes du XXXe congrès de la SELF (pp. 272-279). Biarritz : France.

Weill-Fassina, A., \& Pastré P. (2004) Les compétences professionnelles et leur développement. In : P. Falzon (Ed.) Traité d'ergonomie. Paris : PUF.

\section{RESÚMENES}

Desde hace unos años, las relaciones de servicio, son estudiadas desde diferentes perspectivas y disciplinas, tales como, economía, ergonomía, etnología, gestión, psicología y sociología, entre otras. No obstante, las relaciones de servicio de intervención psico-educativa-sanitarias, son aun desconocidas. Identificar las condiciones bajo las cuales estas se desarrollan, así como las competencias que éstas exigen en los operadores, parece importante desde un punto de vista científico, social y gremial. En definitiva, el objetivo que estos trabajadores y trabajadoras persiguen es de asegurar el cuidado del beneficiario y sobre todo favorecer su evolución, en un contexto de regulación permanente.

Desde há uns anos, as relações de serviço têem sido estudadas em diferentes perspectivas e disciplinas, tais como a economia, a ergonomia, a etnologia, a gestão, a psicologia e sociologia, entre outras. Mas, apesar disso, as relações de serviço de intervenção psico-educativa-sanitaria são ainda desconhecidas. Identificar as condições nas quais estas se desenvolvem, assim como as competências que estas exigem aos operadores, parece importante de um ponto de vista científico, social e profissional. $\mathrm{O}$ objectivo que estes trabalhadores e trabalhadoras perseguem é o de assegurar o cuidado do beneficiário e, sobretudo, favorecer a sua evolução num contexto de regulação permanente.

Depuis quelques années, les relations de service sont étudiées par différentes disciplines, notamment en économie, ergonomie, ethnologie, gestion, psychologie ou sociologie. Malgré cela, les relations de service d'intervention psycho-educativessanitaires restent encore méconnues. Identifier les conditions dans lesquelles elles s'opèrent et les compétences qu'elles impliquent parait important d'un point de vue scientifique, social et professionnel. En fait, le but que poursuivent ces travailleurs et travailleuses est d'assurer la prise en charge du bénéficiaire et surtout de favoriser son évolution dans un contexte de régulation permanente.

During the last few years, service relations have been investigated by economists, ergonomists, ethnologists, managers, psychologists and sociologists. However, work done in 
psychoeducational-care services remains quite unknown. So, for scientific and social reasons as well as for occupational practice, it is very important to identify the skills involved and the specific work conditions. The operators' aim is to take care of beneficiaries and to help them to evolve in a context of constant regulation.

ÍNDICE

Mots-clés: relations de service, activité psycho-éducative-sanitaire

Palavras-chave: relações de serviço, actividade psico-educativa-sanitária

Keywords: service relations, psycho-educative care activity

Palabras claves: relación de servicio, actividad psico-educativa-sanitaria

\section{AUTOR}

\section{RAFAEL GONZÁLEZ}

Docente-investigador en Ergonomía y Neurociencias del Trabajo - Instituto de Altos Estudios en Salud Pública "Dr. Arnoldo Gabaldon" Ministerio de Salud C.P. : 4739, Zone Postale : 2101-A, República de Venezuela Maracay 803ra@cantv.net | gonzal@cnam.fr 